\title{
Right amygdalar and temporofrontal activation during autobiographic, but not during fictitious memory retrieval
}

\author{
Hans J. Markowitsch ${ }^{\mathrm{a}, *}$, Alexander Thiel ${ }^{\mathrm{b}}$, \\ Mechthild Reinkemeier ${ }^{\mathrm{a}}$, Josef Kessler ${ }^{\mathrm{c}}$, \\ Adem Koyuncu $^{\mathrm{c}}$ and Wolf-Dieter Heiss ${ }^{\mathrm{b}, \mathrm{c}}$ \\ a Physiological Psychology, University of Bielefeld, \\ D-33501 Bielefeld, Germany \\ ${ }^{\mathrm{b}}$ Clinic of Neurology, University of Cologne, Cologne, \\ Germany \\ ${ }^{\mathrm{c}}$ Max-Planck-Institute for Neurological Research, \\ Cologne, Germany
}

\begin{abstract}
What distinguishes the recall of real-life experiences from that of self-created, fictitious emotionally laden information? Both kinds of information belong to the episodic memory system. Autobiographic memories constitute that part of the episodic memory system that is composed of significant life episodes, primarily of the distant past. Functional imaging was used to study the neural networks engaged in retrieving autobiographic and fictitious information of closely similar content. The principally activated brain regions overlapped considerably and constituted temporal and inferior prefrontal regions plus the cerebellum. Selective activations of the right amygdala and the right ventral prefrontal cortex (at the level of the uncinate fascicle interconnnecting prefrontal and temporopolar areas) were found when subtracting fictitious from autobiographic retrieval. Furthermore, distinct foci in the left temporal lobe were engaged. These data demonstrate that autobiographic memory retrieval uses (at least in nonbrain damaged individuals) a network of right hemispheric ventral prefrontal and temporopolar regions and left hemispheric lateral temporal regions. It is concluded that it is the experiential character, its special emotional infiltration and its arousal which distinguishes memory of real-life from that of fictitious episodes. Consequently, our results point to the engagement of a bi-hemispheric network in which the right temporo-prefrontal hemisphere is likely to be responsible for the affective/arousal side of information retrieval and the lefthemispheric temporal gyrus for its engram-like representation. Portions of the neural activation found during retrieval might, however, reflect re-encoding processes as well.
\end{abstract}

Keywords: Episodic memory, ecphory, positron emission to- mography, emotion, uncinate fascicle, amygdala, autobiographical memory, fictitious memory, retrieval

\section{Introduction}

Philosophers declare personal identity as dependent on personal memories and sometimes propose Gedankenexperiments in which the normal coincidence between 'experiencing' and 'remembering' persons is dissolved $[47,51,56]$. Many behavioral neuroscientists distinguish between a number of memory subsystems of which episodic memory is that subsystem which is most vulnerable to certain kinds of brain damage, oriented towards the past, probably unique to human beings, and accompanied by "autonoetic" conscious awareness [60,61]. ('Autonoetic awareness' means self-awareness, or consciously reflected thoughts.) A subcategory of episodic memories are the autobiographic ones, which have been stored for years or even decades. Their retrieval may be selectively impaired in patients with focal retrograde episodic amnesia. These patients frequently have predominantly right temporopolar-prefrontal damage $[8,24,26,34,37]$. They are able to acquire new information after their brain damage, most likely due to the preservation of medial temporal structures. This information is usually acquired in an emotionless, neutral or "semantic" manner [32]. This independence between information acquisition and information retrieval has also been confirmed with functional imaging in normal subjects [16]. (The reverse condition with a dominant lefthemispheric focus in the temporo-frontal region leads

${ }^{*}$ Corresponding author: Hans J. Markowitsch, Physiological Psychology, University of Bielefeld, P.O. Box 1001 31, D-33501 Bielefeld, Germany. Tel.: +49 521106 4487; Fax: +49 521106 6049; E-mail: hjmarkowitsch@uni-bielefeld.de. 
to a selective inability to retrieve information from the semantic past $[3,35])$.

Consequently, it was hypothesized that the right ventral branch of the uncinate fascicle, interconnecting the temporopolar and inferior prefrontal cortex, is an important link for this regional combination and that this structural conglomerate - which includes the amygdala - is necessary for successful autobiographic memory retrieval [30,33] (Fig. 1(A)). This form of retrieval has been named 'ecphory' to indicate the fact that episodes from the personal past are usually retrieved within a specific context provided by the environment $[55,60]$.

Fink et al. [16] asked subjects to remember affectladen memory episodes of their own past and compared the resulting brain activation to that during retrieving recently learned information of another biography, the latter one being much less emotional because not important to the subject. Their findings point to an important difference in the neural activation patterns associated with the retrieval of old autobiographic as opposed to impersonal episodic information of someone else, the latter engaging frontal "retrieval structures", the former temporopolar structures in addition to frontal ones [30].

From this report the following question arises: What will be the difference in brain activation when a given subject retrieves real-life experienced old information in contrast to fictitious, self-generated, but not experienced information of the same nature? Both kinds of information belong to the episodic memory system. Real-life information has been experienced, robustly stored in long-term memory and is affect-laden. Self-created "episodes" also involve the own person and are of an affect-laden nature. They lack, however, the flavor of personal experience, that is, they lack the possible remembering of changed bodily conditions, changed mood states, and reactions of the environments, accompanying the experience. Our hypothesis was that both autobiographic-experienced and self-generated fictitious memories will largely engage the same brain structures. But retrieval of autobiographic episodes will, because they have been selfexperienced in an emotional context, activate emotionsensitive brain regions to a higher degree than fictitious, "self-constructed", information, even if the fictitious episodes are very similar in content to the real-life experienced ones. The higher emotional engagement in retrieving old experienced episodes should be reflected by a special activation of emotion-related brain structures, particularly the amygdala $[7,11,31,36]$.

The comparison of autobiographically experienced old memories with fictitious episodes differs from our previous comparison of autobiographical with impersonal memories [16] in the following ways: The fictitious information was self-generated, created by the tested subject and was likely attributable to the respective individual. It was furthermore closely matched to events that actually had happened. The structure of this kind of information had a very strong affinity to autobiographical true events and, thus, belonged to this category. In the Fink et al. report, on the other hand, subjects listened to events of an alien subject's past. Without reference to the subjects, these events therefore were usually much less emotionally arousing to them and their salience was in general most likely lower than that of the fictitious, but self-generated events.

In order to test our hypothesis we used $\mathrm{H}_{2}^{15} \mathrm{O}$ positron emission tomography and studied the brain activation patterns during the retrieval of fictitious as compared to real-life autobiographic episodes in normal subjects.

\section{Materials and methods}

\subsection{Subjects and procedure}

The study design of Fink et al. [16] was followed in order to test this hypothesis: Eight healthy young male right-handed adults (aged 18-32 years, mean 25.62 years) without previous neurological or psychiatric illnesses or syndromes were employed. Informed consent according to the declaration of Helsinki was obtained from all subjects. They were interviewed with respect to significant personal episodes from their past life (divided into childhood, adolescence, recent past) (condition: AUTOBIOGRAPHIC). Furthermore, subjects were asked to invent or create emotionally arousing episodes of the same affective and interest-provoking character as their real ones. These created episodes should be of a very similar nature as those which actually had happened (condition: FICTITIOUS); subjects were asked to produce faked events from them for the time periods childhood, adolescence, and recent past. Real and fictitious episodes were matched as closely as possible. This was done by instructing the subjects accordingly, by discussing the provided episodes with them, and by selecting those which appeared similar from contents and style. For each of the invented episodes, subjects were asked whether there was indeed no background in real life. (For example, portions of a true and of an invented episode: "When I flew the first time, it was to London, and I was accom- 
A

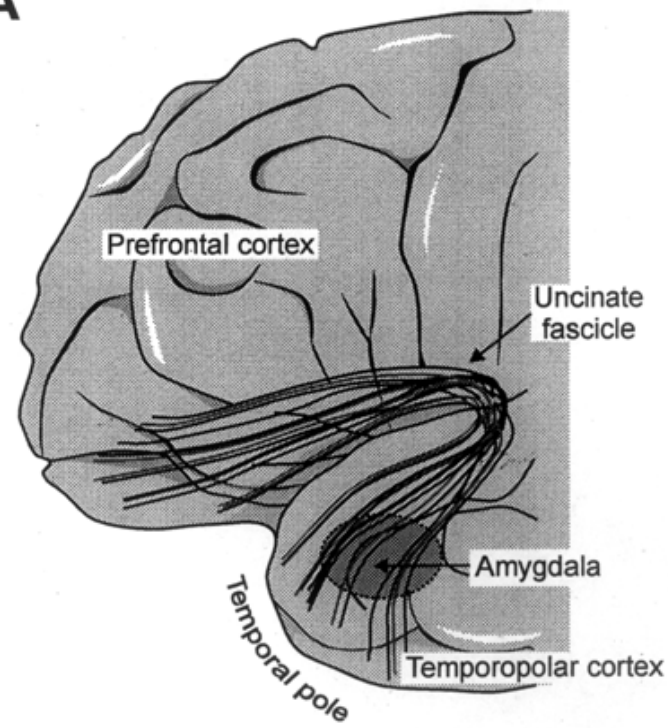

B

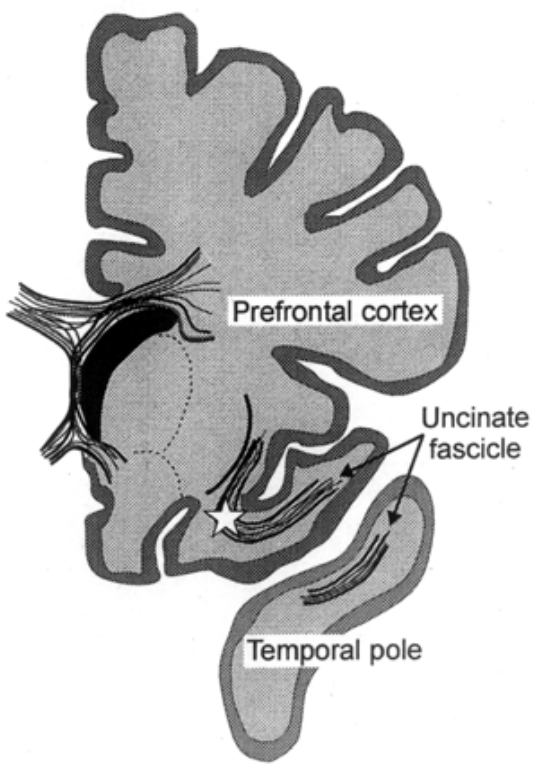

Fig. 1. Sketches of the locus of the (ventral) uncinate fascicle and the amygdala (A) and of the region containing the activity spot in the ventral prefrontal cortex near the uncinate fascicle (B). A: Schematic view of the lateral anterior half of the human cerebral cortex. The course of the ventral branch of the uncinate fascicle, interconnecting the anterior temporal and the ventral prefrontal cortex, and the locus of the amygdala within the anterior medial temporal lobe are shown schematically. B: Coronal section at the level of maximal activation in the region of the uncinate fascicle (star; coordinates: 2616 -12). (After Fig. 74 of Talairach and Tournaux [58]).

panied by my girl friend; my parents did not know." "I drove to Amsterdam with two friends; we bought hashish there which was later detected by the German border guards.")

Both fictitious and true events were discussed with the subjects after production and again (on another day) prior to testing. There was nearly one hour time to think about all events prior to scanning. For each of the episodes a short key sentence was created in the first session which immediately evoked the respective episode. Examples for the true and the faked sentences were: "Marathon run in Saloniki." and "Meeting with Blümchen." (a local pop star). These sentences were used to immediately evoke the respective episode.

As in the previous study of Fink et al. [16] a control or baseline condition (REST) was used in addition.

Up to 24 true (condition: AUTOBIOGRAPHIC) and 24 fictitious episodes (condition: FICTITIOUS) were used while the subjects were placed under ${ }^{15} \mathrm{O}$-positron emission tomography (PET) for scanning their brain (4 sessions per testing condition plus 4 sessions without stimulation ["REST"]) (cf. [16]). For each AUTOBIOGRAPHIC and FICTITIOUS session, episodes were mixed from epochs from childhood to recent past.

The number of episodes used and their character make it likely that, for each subject, a sufficient diver- sity of positive and negative ones was covered; furthermore, individuals stated for the episodes selected that these constituted the ones which to them most clearly and most instantaneously provoked an affective reaction. All subjects were able to successfully retrieve all episodes asked for. There were not exactly, but roughly the same proportion of positive and negative events for a given subject.

\subsection{Positron emission tomographic imaging}

In principal, the study paradigm followed that described in Fink et al. [16]. It involved 12 sequential measurements of relative $\mathrm{rCBF}$ and consisted of one control or baseline condition (REST) in which subjects were studied at rest without auditory or visual stimulation, and two activation conditions (AUTOBIOGRAPHIC: auditory presentation of sentences triggering episodic information of the subjects' own biography; FICTITIOUS: auditory presentation of sentences triggering subject-invented episodic information of a biographical character). The sentences were presented auditorily at a fixed rate of one every 10 seconds; presentation started with i.v. bolus injection and lasted for the entire scanning period. Subjects were instructed to imagine what happened to themselves in the described 
situations under both the AUTOBIOGRAPHIC and the FICTITIOUS condition. The sequence of the presentation of the three conditions was counterbalanced in the following way: AFRRFAARFFRA (A = AUTOBIOGRAPHIC, $\mathrm{F}=$ FICTITIOUS, $\mathrm{R}=$ REST).

The PET measurements of relative CBF were performed on a CTI/Siemens ECAT EXACT HR camera in 3D mode [62] after i.v. bolus injection of $370 \mathrm{MBq}$ of ${ }^{15} \mathrm{O}$-water. Data acquisition started automatically when an activity threshold of $100 \%$ background activity was reached and lasted for $45 \mathrm{~s}$ with a short interscan interval of $6 \mathrm{~min}$ [10]. After correction for scatter and measured attenuation, images were reconstructed to 47 transaxial slices using filtered backprojection and a Hanning filter of cutoff frequency 0.4 cycles per pixel. The resolution of the resulting images was $5.5 \mathrm{~mm}$ (FWHM). Data analysis was performed using SPM 96 [20]. After realignment and spatial normalization to standard stereotactic space [19], images were smoothed applying a Gaussian filter of $16 \mathrm{~mm}$ FWHM. After scaling the images to overall grand mean, t-statistic maps were generated for the contrasts (FICTITIOUS-REST), (AUTOBIOGRAPHICREST), (AUTOBIOGRAPHIC-FICTITIOUS) and (FICTITIOUS-AUTOBIOGRAPHIC) treating global $\mathrm{CBF}$ changes as a covariate. Subsequently t-statistic maps were transformed to $\mathrm{z}$-statistic maps and a threshold level was set to $p<0.01$ (uncorrected). For localizing PET measured CBF changes, magnetic resonance imaging was performed in a $1 \mathrm{~T}$ Magnetom Impact (Siemens Medical Systems, Erlangen, Germany) using a FLASH sequence (flip angle $40^{\circ}$, TR $40 \mathrm{msec}$, TE $15 \mathrm{msec}$ ) producing 64 transaxial T1-weighted slices of $2 \mathrm{~mm}$ thickness in a $256 \times 256$ pixel matrix. Fusion maps of standard magnetic resonance imaging and Zstatistic maps were generated for visualization of results (Figs 2 and 3).

\section{Results}

Autobiographic memory retrieval (AUTOBIOGRAPHIC-REST) under PET-imaging activated mainly regions in the right and left superior temporal gyri, the left middle temporal and inferior prefrontal cortex, and the right cerebellum. Activations were seen in both dorsomedial frontal cortices, the left anterior and posterior cingulate gyrus, the precuneus and the medial thalamus (Fig. 2).

Retrieval of fictitious information [FICTITIOUSREST] engaged the same major activated regions as retrieval of autobiographic information (both superior temporal gyri, left middle temporal and inferior prefrontal cortex, and right cerebellum), plus the left insula. Furthermore, less significant activations were seen in the right posterior hippocampal, right and left prefrontal and anterior cingulate cortex and in the left precuneus (Fig. 2).

The most interesting activations resulted from comparing the two test conditions: Subtracting FICTITIOUS from AUTOBIOGRAPHIC activations revealed distinct activity spots in the right amygdala, uncus and temporopolar regions, in the region of the right ventral prefrontal cortex (at the level of the uncinate fascicle) and in those of the left superior and middle temporal lobe and the anterior cingulate cortex (Figs 1 and 3 ).

Another particularly interesting spot of increased activity in the condition AUTOBIOGRAPHIC-FICTITIOUS was situated in the fundus region (Fig. 3) at the insular limen; this is in that part of the inferior prefrontal cortex which constitutes the bending point of the ventral uncinate fascicle. This fiber bundle establishes a clamp between anterior (prefrontal, orbitofrontal) and posterior (temporal) association cortices which most likely allows a backward and forward propagation of information obtained from the principal left- as well as right-hemispheric storage places along the temporal cortex - another region which showed up selectively in our AUTOBIOGRAPHIC-FICTITIOUS comparison (Fig. 3). Furthermore, the anterior cingulate region was activated as well (Fig. 3).

Finally, the retrieval of fictitious information which had only recently been created (FICTITIOUS-AUTOBIOGRAPHIC), left solely the left precuneus as activated region (coordinates according to Talairach and Tournaux [58]: $-2-7056)$.

\section{Discussion}

The present findings confirmed our previous ones [16] in demonstrating that autobiographic memory retrieval activates a neural net in which the right temporofrontal region is a keystone. Beyond this finding, it demonstrated that the retrieval of real-life as opposed to fictitious information - which for the outside listener appear of comparable content - indeed activates different brain structures: particularly the right uncus and amygdala region, a tiny elongated cortical strip which includes temporal and frontal areas, the anterior cingulate region, and focal spots in the left temporal cortex. 


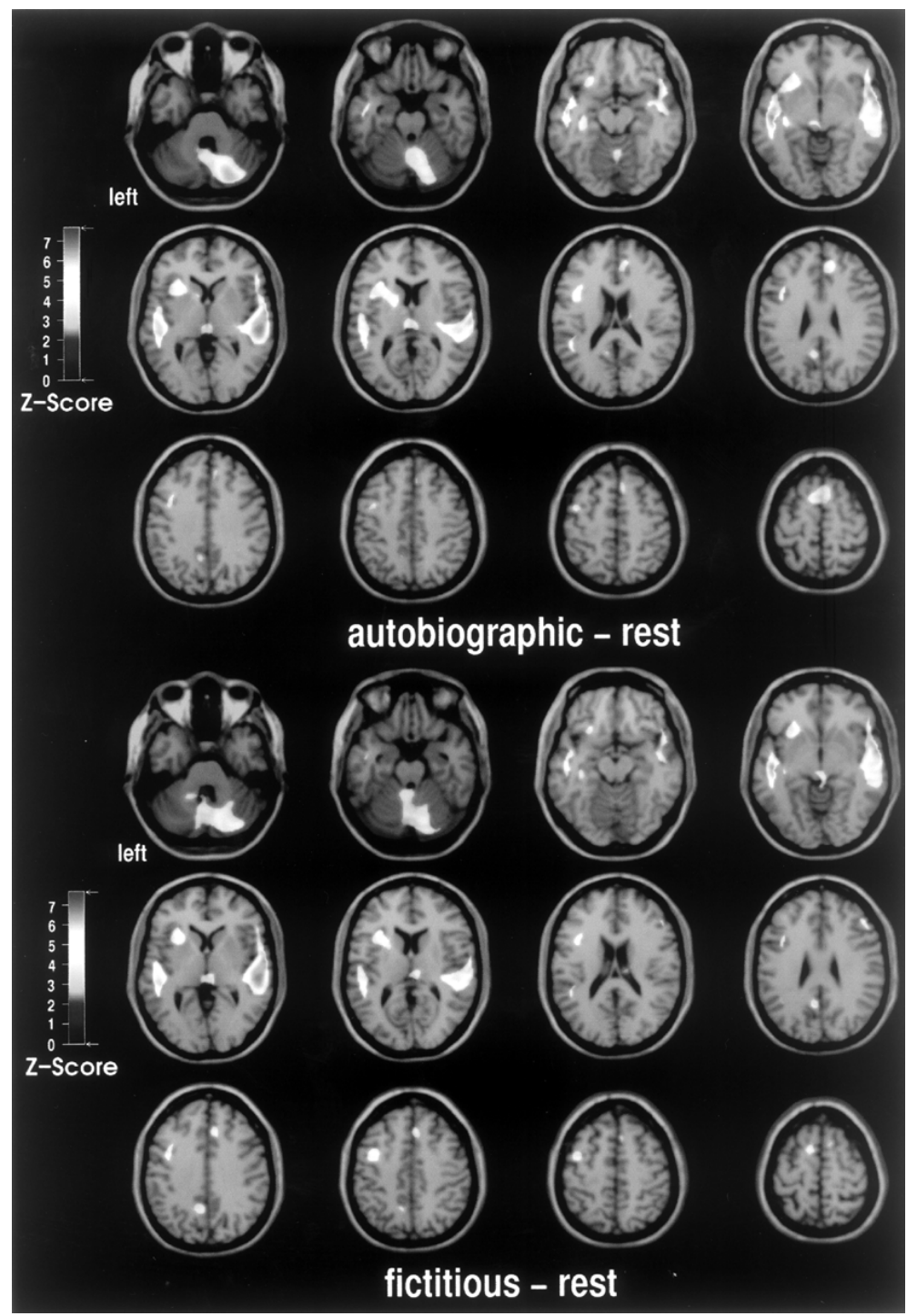

Fig. 2. Striking similarity of the brain activation patterns during affect-laden retrieval of real-life episodes (top; comparison AUTOBIOGRAPHIC-REST) and invented episodes (bottom; comparison FICTITIOUS-REST). The SPM-map is sectioned in transverse planes and is displayed on top of an arbitrary magnetic resonance image that has been normalized spatially to the same anatomical space. The color bars indicate the $\mathrm{Z}$ statistics achieved.

The findings obtained in the conditions AUTOBIOGRAPHIC-REST and FICTITIOUS-REST are largely in conformity with our hypothesis and previous findings $[16,38]$ and will not be commented here. Of considerable interest, novelty and importance are, however, the results obtained from comparing the two testing conditions (AUTOBIOGRAPHIC-FICTITIOUS; FICTITIOUS-AUTOBIOGRAPHIC): Distinct scenes of positively or negatively emotionally laden episodes had to be retrieved and imagined. The scenes differed only in being true, that is personally experienced and stored for years, or in being fictitious that is, not having been experienced. The real-life episodes furthermore were most likely felt and re-experienced with deep, intimate affect, which is represented in the activation of the amygdala and the surrounding temporo-polar region (Figs 1 and 3). The necessity of this region for autobiographic memory retrieval had been postulated already previously [31], both from neuroimaging results in human subjects $[16,39]$ and from patient data $[8$, $24,26,28,34,37]$, but only this direct test of retrieval of autobiographic versus fictitious information, which for 


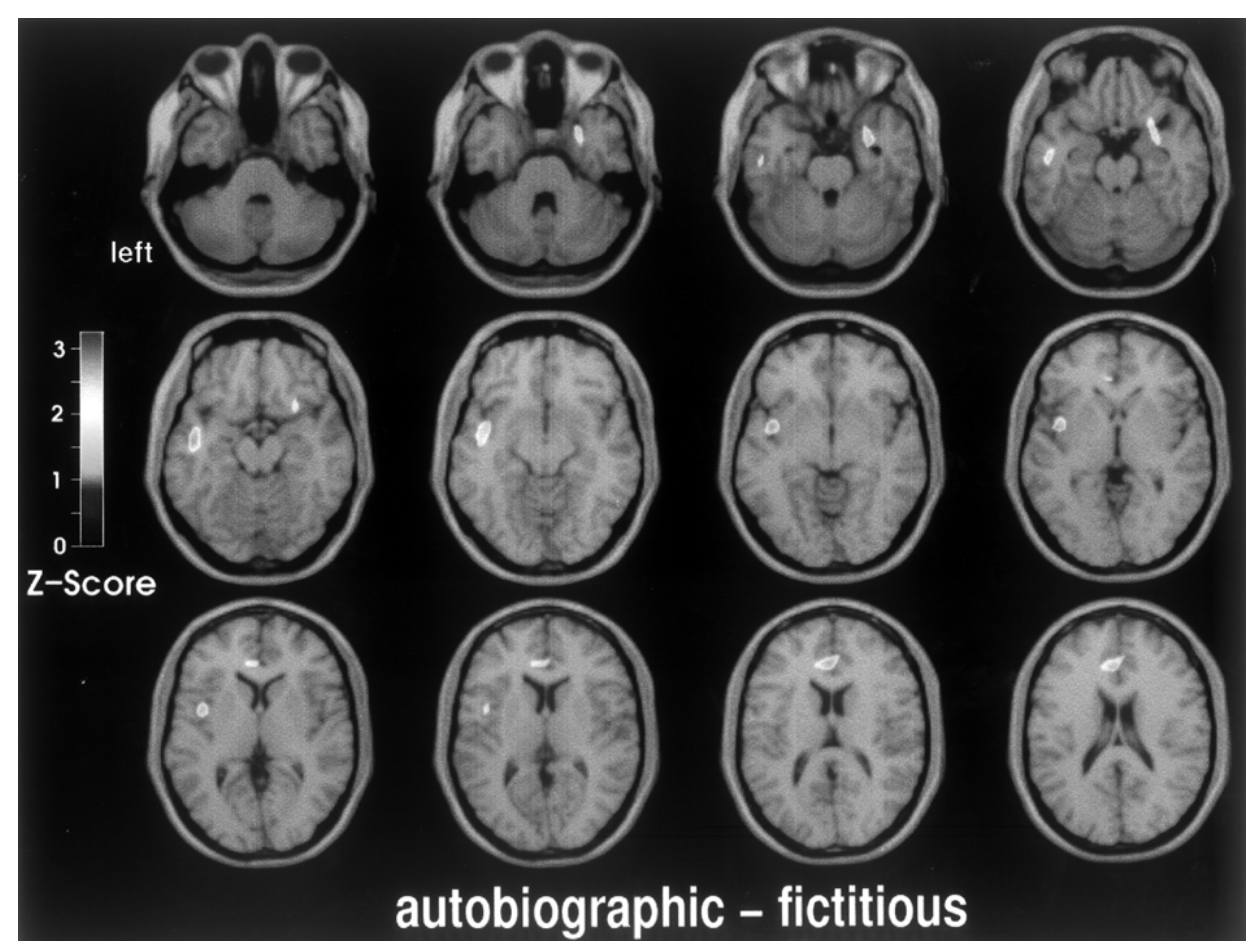

Fig. 3. Functional anatomy of temporal activations during affect-laden autobiographic memory retrieval (comparison AUTOBIOGRAPHIC-FICTITIOUS). The SPM-map is sectioned in transverse planes and is displayed on top of an arbitrary magnetic resonance image that has been normalised spatially to the same anatomical space. The color bar indicates the $\mathrm{Z}$ statistics achieved. The activations are found in the regions of the right amygdala and uncus, including the place where the uncinate fascicle passes on its way between the anterior temporal and the inferior prefrontal cortex. In the first row, the 1st, 2nd and 3rd sections (from left to right) show amygdaloid activation spots: 300 - 20 ; 260 -26 , the 4 th section the activation spot in the ventral prefrontal cortex near the uncinate fascicle: $2616-12$. Activations in the left middle and superior temporal lobe are visible in the 3rd and 4th section of the top row, in all sections of the medial row and in the 1st and 2nd section of the bottom row; activation spots: $-50-12-10 ;-44210 ;-46-2-2$. The activations in the anterior cingulate cortex can be seen in the 4 th section of the middle row and in all bottom sections; activation spots: $-43424 ;-6368$.

the listening outsider is of an indistinguishable character (affect-laden episodes), provided direct evidence for right amygdalar engagement during the retrieval of personal old memories. The smallness of the z-scores of the regions shown in Fig. 3 most likely reflects the otherwise high similarity of the retrieval process for these two kinds of episodic memory.

There may be other interpretations for the differential activation during autobiographic as opposed to fictitious memory. Firstly, it can be argued that the two kinds of information reflect different states of arousal; secondly, the differential activation might be related to differing time-scales (information stored for years vs. information stored recently). The arousal-related interpretation would in principle be compatible with our view as arousal and emotion are highly interrelated. An interpretation based purely on the closeness of the retrieved information to the present time appears, however, unlikely as (a) both kinds of information had recently been retrieved and (b) usually the more recent information is accompanied by a higher degree of emotion, arousal and detail than information from the distant past (This should lead to an activation of those limbic regions which in fact are now engaged during the retrieval or ecphory of autobiographic information).

Factors contributing to the differential activation of fictitious as opposed to true memories might also be seen in differences in their imageability and in differences in the detail of the information retrieved and reencoded. While these factors may be of quantitative influence, we do not think that they had a major influence on the differential activations, as the instructions for production (and the outcome provided by the subjects) revealed great detail and fantasy and as the subjects all were very motivated university students.

\subsection{Amygdaloid and temporofrontal regions}

An important finding is the selective right hemispheric activation in the amygdaloid and tempo- 
rofrontal regions during autobiographic retrieval. A number of different roles in memory processing have been attributed to the amygdala [31]. The variation in these roles depends to a considerable degree on the task situations investigated. There are presently, however, congruent findings both from neuropsychological results $[1,7,36]$ and from electrophysiological recordings [52-54] which point to a prominent role of the amygdala in the processing of explicit memories, particularly as these involve awareness and real-life emotional events [4]. Mori et al. [42] found in Alzheimer's patients a correlation between normalized amygdaloid volume and extent of emotional memory retrieval (reporting episodes of the 1995 Kobe earthquake), while generalized knowledge of the earthquake was unrelated to amygdalar volume.

Right amygdalar activation during autobiographic memory retrieval was found in our previous PET study as well [16]. Damage to the right temporo-frontal region which included the right but not the left amygdala, resulted in a selective failure to retrieve autobiographic episodes [8], and thus destroyed the autonoetic awareness $[60,61]$ of oneself which is necessary for re-experiencing past events as part of one's own identity [28]. Together with our present results, these findings point to a major role of the right amygdala in creating the affect-laden, lively nature of our wellpreserved biography, and therefore in autobiographic memory retrieval.

The right temporofrontal junction region (which is interconnected via the ventral branch of the uncinate fascicle) has been repeatedly proposed to be necessary for effective autobiographic memory retrieval [26,2830]. The clear activation of this region of the right hemisphere in our present study strongly reinforces this view which up to now came primarily from patient data. A recently published study reports a patient who had a traumatic focal injury, disrupting the right uncinate fascicle selectively [28]. As a consequence of the accident he remained unable to retrieve old autobiographic memories and showed a severe reduction in his self-awareness.

From other patient data it is known that damage to the temporofrontal region, particularly of the right hemisphere, may lead to delusional distortions, confabulatory tendencies, or even reduplicative paramnesia [2, 40]. A meta-analysis of 488 cases with Capgras syndrome found in one third of the patients a neurological etiology, usually either in the right frontal or the left temporal lobe [57]. (Note that our results showed activation pattern in the left temporal lobe as well.)
Recently, Dolan et al. [14] studied emotional episodic memory retrieval with PET. In addition to a right anterior temporal activation, they observed a left amygdaloid response during the actual retrieval of emotional items. Their material, however, were affectladen, but impersonal pictures and had been studied only just prior to scanning; it therefore differs considerably from the imaged complex scenes of our subjects that were self-experienced or self-created and, thus, had a personal reference. With respect to the encoding of emotional information, Hamann et al. [23], in another PET study, found bilateral amygdala activation during encoding. As each retrieval of information leads to reencoding as well, our activation pattern might (perhaps in part) also be related to re-encoding processes.

\subsection{Cingulate cortex}

The anterior cingulate cortex is frequently found to be increased in activity in blood flow activation studies [48]. Its role in the present setting is probably multiple: There are likely engagements related to anticipation, arousal, visual imagery or re-experiencing $[6$, 16-18,44,59] and to hypothesis testing and response selection [16] - processes necessary for a successful retrieval of already experienced information.

Furthermore, the anterior cingulate cortex is of course a keystone in the emotion-processing circuitry of the limbic system. Lane et al. [27] studied the neural correlates of emotion in healthy volunteers, using an 'emotional awareness scale' in combination with PET. They found that film- and recall-induced emotion overlapped significantly with activations of the anterior cingulate cortex. Devinsky et al. [13] pointed to the involvement of the anterior cingulate cortex in components of affect, motivation, and cognition and stressed its extensive connections with the amygdala. The combined activation of the amygdala and the anterior cingulate cortex, as seen in our study, seems to represent the affect-related components of retrieving/ecphorizing (and re-encoding) episodes from the (distant) past.

\subsection{Temporal cortex}

The selective engagement of left-hemispheric regions of the middle and superior temporal gyrus was not predicted. In part it may reflect the engagement of regions during retrieval which are also (and most likely even primarily) associated with the actual storage places of the engrams $[9,22,41,49]$. From the PET results of Mummery et al. [43] it can be proposed that 
the more complex the generation of verbal information becomes, the more it engages the temporal lobes.

The activated regions of the left temporal cortex possibly provide conceptual dimensions of an imagined episode, experienced in reality. In line with this idea are the results of Schacter et al. [51]. These authors compared brain regions activated by veridical recognition of printed words (heard several minutes earlier) and illusory recognition of printed words (not heard earlier). They found that activation in a left temporoparietal region, which previously had been implicated in the retention of auditory information, distinguished accurate from illusory recognition memory.

It should be noted that the unilateral, left temporal activation was found after subtracting FICTITIOUS from AUTOBIOGRAPHIC activations, and not by subtracting REST from AUTOBIOGRAPHIC activations. While the latter provided bi-hemispheric temporal activations, including the hippocampal region (cf. Fig. 2), the former led to the much more selective activation pattern, which, with respect to the temporal lobes, gave only very restricted left temporal activations (Fig. 3). Compared to our previous report [16], this activation pattern is much narrower and can be interpreted as reflecting the great similarity in the ecphorized scenes, differing in fewer dimensions than the autobiographic versus impersonal episodic events in the Fink et al. [16] study. Most likely, only the experience of having a wider and more intense imaginative conception with the self-experienced compared to selfcreated images, reflects the differential activation in the AUTOBIOGRAPHIC-FICTITOUS comparison.

\subsection{Precuneus}

The precuneus has been termed "the mind's eye" [17, 18 ] because of its role in memory-related imagery [25]. This is most likely the reason for its activation in the present study when subtracting autobiographic from fictitious retrieval, as fictitious material needs more imagination for successful retrieval (or ecphory) than real-life experienced material. It can be assumed that despite their recent creation and retrieval, imagination of fictitious material for retrieval is more of a creative, fantasy requiring and constructive act than is the retrieval of events that actually had happened in previous life.

\section{Conclusions}

Our present findings of selective activations in the regions of the amygdala, of the cortex next to the uncinate fascicle, the anterior cingulate cortex and the middle and superior temporal cortex all point to a specific network of regions involved in retrieving old autobiographic memories. It seems that an array of structures of the extended limbic system (inferior prefrontal/orbitofrontal cortex, amygdala, anterior cingulate cortex) $[45,46]$ interacts with storage places in the temporal cortex [41] to retrieve affect-laden autobiographic episodes.

Consequently, travelling mentally back in time and becoming consciously aware of having experienced the retrieved information most likely engages a more complex limbic-neocortical network than does the simple ecphory of self-created information without a real-life background. In the words of Brown ([5, p. 140]), "a limbic transition in the outward development of the mental state", "a relation of feeling, to the personal history and the immediate past" constitute the selfconcept. Our study demonstrates the existence of a functional anatomical network for his proposal.

\section{Acknowledgements}

The authors thank E. Tulving for helpful comments on an earlier draft of the manuscript and A. Reinhard for help with the subjects' interviews. Supported by the Deutsche Forschungsgemeinschaft (Ma 795/24).

\section{References}

[1] R. Adolphs, H. Damasio, D. Tranel and A.R. Damasio, Cortical systems for the recognition of emotion in facial expressions, Journal of Neuroscience 16 (1996), 7678-7687.

[2] M.P. Alexander and D.R. Stuss, On Capgras syndrome: a reduplicative phenomenon, Journal of Psychosomatic Research 44 (1998), 637-639.

[3] W.B. Barr, Examining the right temporal lobe's role in nonverbal memory, Brain and Cognition 35 (1997), 26-41.

[4] M.M. Bianchin and C.W. Spanis, Involvement of the amygdala in explicit memories, Neurology 51 (1998), 1520.

[5] J.W. Brown, Neuropsychology and the self-concept, Journal of Nervous and Mental Disease 187 (1999), 131-141.

[6] R. Cabeza and L. Nyberg, Imaging cognition: An empirical review of PET studies with normal subjects, Journal of Cognitive Neurosciences 9 (1997), 1-26.

[7] L. Cahill, R. Babinsky, H.J. Markowitsch and J.L. McGaugh, Involvement of the amygdaloid complex in emotional memory, Nature 377 (1995), 295-296. 
[8] P. Calabrese, H.J. Markowitsch, H.F. Durwen, B. Widlitzek, M. Haupts, B. Holinka and W. Gehlen, Right temporofrontal cortex as critical locus for the ecphory of old episodic memories, Journal of Neurology, Neurosurgery, and Psychiatry 61 (1996), 304-310.

[9] W.R. Chen, S. Lee, K. Kato, D.D. Spencer, G.M. Shepherd and A. Williamson, Long-term modifications of synaptic efficacy in the human inferior and middle temporal cortex, Proceedings of the National Academy of Sciences of the USA 93 (1996), 8011-8015.

[10] J. Chmielowska, R.C. Coghill, J.-M. Maisog, R.E. Carson, P. Herscovitch, M. Honda, R. Chen and M Hallett, Positron emission tomography $\left[{ }^{15} \mathrm{O}\right]$ water studies with short interscan intervala for single-subject and group analysis: influence of background subtraction, Journal of Cerebral Blood Flow and Metabolism 18 (1998), 433-443.

[11] R.J. Davidson and W. Irwin, The functional neuroanatomy of emotion and affective style, Trends in Cognitive Sciences 3 (1999), 11-21.

[12] E. De Renzi, M. Liotti and P. Nichelli, Semantic amnesia with preservation of autobiographic memory. A case report, Cortex 23 (1987), 575-597.

[13] O. Devinsky, M.J. Morrell and B.A. Vogt, Contributions of anterior cingulate cortex to behaviour, Brain 118 (1995), 279306.

[14] R.J. Dolan, R. Lane, P. Chua and P. Fletcher, Dissociable temporal lobe activations during emotional episodic memory retrieval, NeuroImage 11 (2000), 203-209.

[15] R. Elliott and R.J. Dolan, Activation of different anterior cingulate foci in association with hypothesis testing and response selection, NeuroImage 8 (1998), 17-29.

[16] G.R. Fink, H.J. Markowitsch, M. Reinkemeier, T. Bruckbauer, J. Kessler and W.-D. Heiss, Cerebral representation of one's own past: neural networks involved in autobiographical memory, Journal of Neuroscience 16 (1996), 4275-4282.

[17] P.C. Fletcher, C.D. Frith, S.C. Baker, T. Shallice, R.S.J. Frackowiak and R.J. Dolan, The mind's eye: Precuneus activation in memory-related imagery, NeuroImage 2 (1995), 195-200.

[18] P.C. Fletcher, T. Shallice, C.D. Frith, R.S. Frackowiak and R.J. Dolan, Brain activity during memory retrieval: the influence of imagery and semantic cueing, Brain 119 (1996), 1587-1596.

[19] K.J. Friston, J. Ashburner, C.D. Frith, J.-B. Poline, J.D. Heather and R.S. Frackowiak, Spatial registration and normalization of images, Human Brain Mapping 2 (1995), 1-25.

[20] K. Friston, A.P. Holmes, K.J. Worsley, J.B. Poline, C.D. Frith and R.S. Frackowiak, Statistical parametric maps in functional imaging: A general linear approach, Human Brain Mapping 2 (1995), 189-210.

[21] O. Ghaem, E. Mellet, F. Crivello, N. Tzourio, B. Mazoyer, A. Berthoz and M. Denis, Mental navigation along memorized routes activates the hippocampus, precuneus, and insula, Neuroreport 8 (1997), 739-744.

[22] M.M. Haglund, A. Ojemann, T.W. Schwartz and E. Lettich, Neuronal activity in human lateral temporal cortex during serial retrieval from short-term memory, Journal of Neuroscience 14 (1994), 1507-1515.

[23] S.B. Hamann, T.D. Ely, S.T. Grafton and C.D. Kilts, Amygdala activity related to enhanced memory for pleasant and aversive stimuli, Nature Neuroscience 2 (1999), 289-293.

[24] N. Kapur, Focal retrograde amnesia in neurological disease: A critical review, Cortex 29 (1993), 217-234.

[25] B.J. Krause, D. Schmidt, F.M. Mottaghy, U. Halsband, H. Herzog, L. Tellmann and H.W. Müller-Gärtner, Episodic retrieval activates the precuneus irrespective of the imagery con- tent of word pair associates. A PET study, Brain 122 (1999), 255-263.

[26] N. Kroll, H.J. Markowitsch, R. Knight and D.Y. von Cramon, Retrieval of old memories - the temporo-frontal hypothesis, Brain 120 (1997), 1377-1399.

[27] R.D. Lane, E.M. Reiman, B. Axelrod, L.-S. Yun, A. Holmes and G.E. Schwartz, Neural correlated of levels of emotional awareness: Evidence of an interaction between emotion and attention in the anterior cingulate cortex, Journal of Cognitive Neuroscience 10 (1998), 525-535.

[28] B. Levine, S.E. Black, R. Cabeza, M. Sinden, A.R. McIntosh, J.P. Toth, E. Tulving and D.T. Stuss, Episodic memory and the self in a case of isolated retrograde amnesia, Brain 121 (1998), 1951-1973.

[29] H.J. Markowitsch, Which brain regions are critically involved in the retrieval of old episodic memory? Brain Research Reviews 21 (1995), 117-127.

[30] H.J. Markowitsch, The functional anatomy of episodic memory retrieval, Trends in Neurosciences 20 (1997), 557-558.

[31] H.J. Markowitsch, Differential contribution of the right and left amygdala to affective information processing, Behavioural Neurology 11 (1998/99), 233-244.

[32] H.J. Markowitsch, Functional neuroimaging correlates of functional amnesia, Memory 7 (1999), 561-583.

[33] H.J. Markowitsch, Memory and amnesia, in: Principles of cognitive and behavioral neurology, M.-M. Mesulam, ed., Oxford University Press, New York, NY, 2000, pp. 257-293.

[34] H.J. Markowitsch, P. Calabrese, J. Liess, M. Haupts, H.F. Durwen and W. Gehlen, Retrograde amnesia after traumatic injury of the temporo-frontal cortex, Journal of Neurology, Neurosurgery, and Psychiatry 56 (1993), 988-992.

[35] H.J. Markowitsch, P. Calabrese, H. Neufeld, W. Gehlen and H.F. Durwen, Retrograde amnesia for famous events and faces after left fronto-temporal brain damage, Cortex 35 (1999), 243-252.

[36] H.J. Markowitsch, P. Calabrese, M. Würker, H.F. Durwen, J. Kessler, R. Babinsky, D. Brechtelsbauer, L. Heuser and W. Gehlen, The amygdala's contribution to memory - A PETstudy on two patients with Urbach-Wiethe disease, NeuroReport 5 (1994), 1349-1352.

[37] H.J. Markowitsch and K. Ewald, Right-hemispheric frontotemporal injury leading to severe autobiographical retrograde and moderate anterograde episodic amnesia, Neurologie Psychiatry and Brain Sciences 5 (1997), 71-78.

[38] H.J. Markowitsch, G.R. Fink, A.I.M. Thöne, J. Kessler and W.D. Heiss, Persistent psychogenic amnesia with a PET-proven organic basis, Cognition and Neuropsychiatry 2 (1997), 135158.

[39] H.J. Markowitsch, A. Thiel, J. Kessler and W.-D. Heiss, Ecphorizing semi-conscious episodic information via the right temporopolar cortex - a PET study, Neurocase 3 (1997), 445449.

[40] M.-M. Mesulam, Notes on the cerebral topography of memory and memory distortion: A neurologist's perspective, in: Memory distortion, D.L. Schacter, ed., Harvard University Press, Cambridge, MA, 1995, pp. 379-385.

[41] Y. Miyashita, M. Kameyama, I. Hasegawa and T. Fukushima, Consolidation of visual associative long-term memory in the temporal cortex of primates, Neurobiology of Learning and Memory 70 (1998), 197-211.

[42] E. Mori, M. Ikeda, N. Hirono, H. Kitagaki, T. Imamura and T. Shimomura, Amygdalar volume and emotional memory in Alzheimer's disease, American Journal of Psychiatry 156 (1999), 216-222. 
[43] C.J. Mummery, K. Patterson, J.R. Hodges and R.J.S. Wise, Generating 'tiger' as an animal name or a word beginning with T: differences in brain activation, Proceedings of the Royal Society of London B 263 (1996), 989-995.

[44] S. Murtha, H. Chertkow, M. Beauregard, R. Dixon and A. Evans, Anticipation causes increased blood flow to the anterior cingulate cortex, Human Brain Mapping 4 (1996), 103-112.

[45] W.J.H. Nauta, Expanding borders of the limbic system concept, in: Functional neurosurgery, T. Rasmussen and R. Marino, eds, Raven Press, New York, 1979, pp. 7-23.

[46] R. Nieuwenhuys, The greater limbic system, the emotional motor system and the brain, in: The emotional motor system, (Progress in Brain Research, Vol. 107), G. Holstege, R. Bandler and C.B. Saper, eds, Elsevier, Amsterdam, 1996, pp. 551580.

[47] D. Parfit, Reasons and persons, Clarendon Press, Oxford, 1984.

[48] T. Paus, L. Koski, Z. Caramanos and C. Westbury, Regional differences in the effects of task difficulty and motor output on blood flow response in the human anterior cingulate cortex: a review of 107 PET activation studies, NeuroReport 9 (1998), R37-R47.

[49] K. Sakai and Y. Miyashita, Visual imagery: an interaction between memory retrieval and focal attention, Trends in Neurosciences 17 (1994), 287-289.

[50] D.L. Schacter, E. Reiman, T. Curran, L.S. Yun, D. Bandy, K.B. McDermott and H.L. Roediger, III, Neuroanatomical correlates of veridical and illusory recognition memory: evidence from positron emission tomography, Neuron 17 (1996), 267274.

[51] M. Schechtman, Personhood and personal identity, Journal of Philosophy 87 (1990), 72-92.

[52] M. Seeck, N. Mainwaring, J. Ives, H. Blume, D. Dubuisson, R. Cosgrove, M.-M. Mesulam and D.L. Schomer, Differential neural activity in the human temporal lobe evoked by faces of family members and friends, Annals of Neurology 34 (1993), 369-372.

[53] M. Seeck, D. Schomer, N. Mainwaring, J. Ives, D. Dubuisson, H. Blume, R. Cosgrove, B.J. Ransic and M.-M. Mesulam, Selectively distributed processing of visual object recognition in the temporal and frontal lobes of the human brain, Annals of Neurology 37 (1995), 538-545.

[54] M. Seeck, N. Mainwaring, M.D. Cosgrove, H. Blume, D. Dubuisson, M.-M. Mesulam and D.L. Schomer, Neurophysiologic correlates of implicit face memory in intracranial visual evoked potentials, Neurology 49 (1997), 1312-1316.

[55] R. Semon, Die Mneme als erhaltendes Prinzip im Wechsel des organischen Geschehens, Wilhelm Engelmann, Leipzig, 1904.

[56] S. Shoemaker and R. Swinburne, Personal identity, Basil Blackwell, Oxford, 1984.

[57] S.F. Signer, Capgras syndrome and delusions of reduplication in neurologic disorders, Neuropsychiatry Neuropsychology and Behavioral Neurology 5 (1992), 138-142.

[58] J. Talairach and P. Tournaux, Co-Planar stereotaxic atlas of the human brain, Thieme, Stuttgart, 1988.

[59] S.F. Taylor, I. Liberzon, L.M. Fig, L.R. Decker, S. Minoshima and R.A. Koeppe, The effect of emotional content on visual recognition memory: a PET activation study, Neuroimage $\mathbf{8}$ (1998), 188-197.

[60] E. Tulving, Elements of episodic memory, Oxford University Press, Oxford, 1983.

[61] E. Tulving and H.J. Markowitsch, Episodic and declarative memory: Role of the hippocampus, Hippocampus 8 (1998), 198-204.

[62] K. Wienhard, M. Dahlborn, L. Eriksson, C. Michel, T. Bruckbauer, U. Pietrzyk and W.D. Heiss, The ECAT EXACT HR: Performance of a new high resolution positron scanner, Journal of Computer Assisted Tomography 181 (1994), 110-118. 


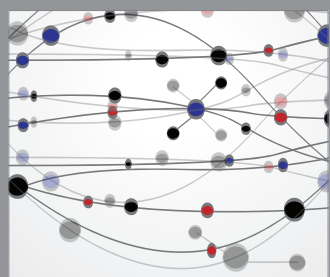

The Scientific World Journal
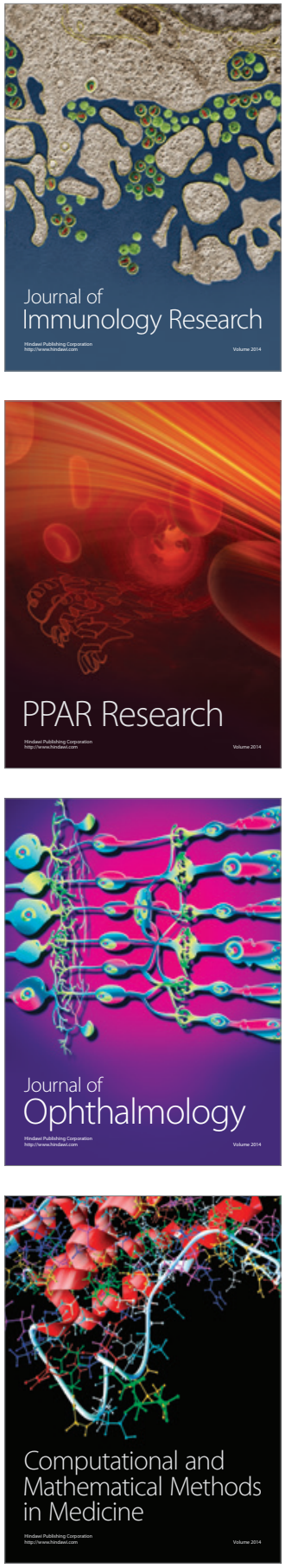

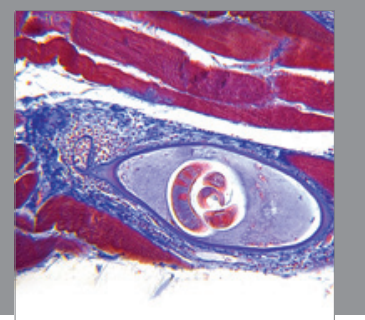

Gastroenterology

Research and Practice
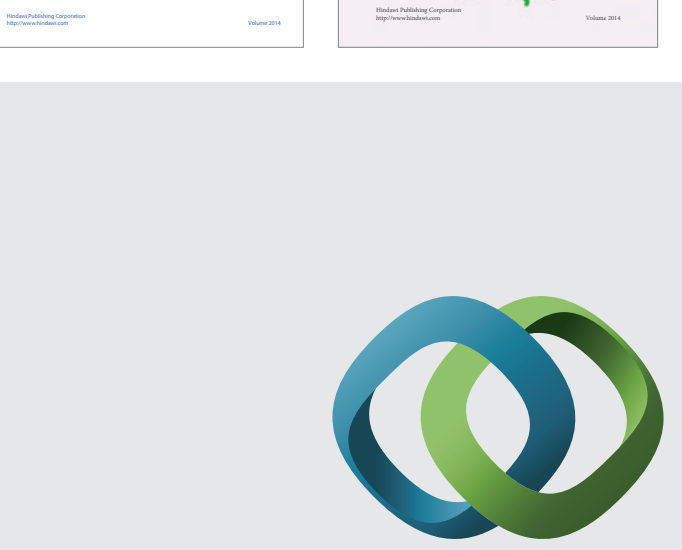

\section{Hindawi}

Submit your manuscripts at

http://www.hindawi.com
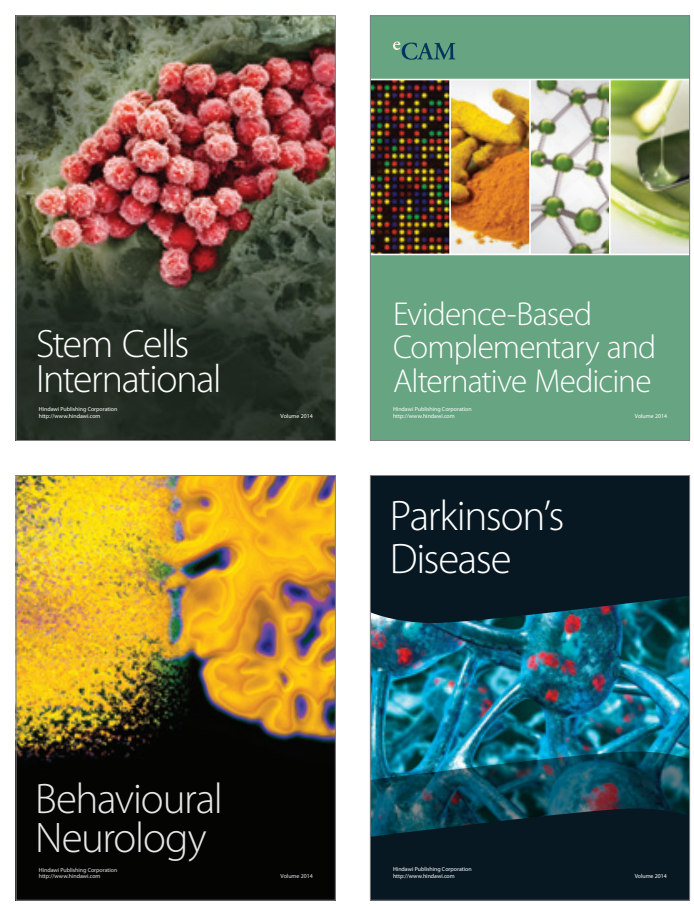

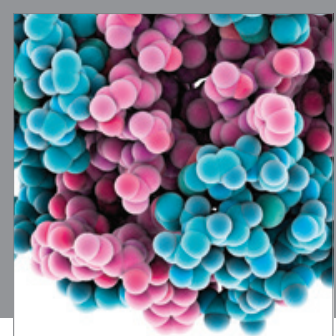

Journal of
Diabetes Research

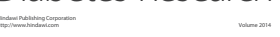

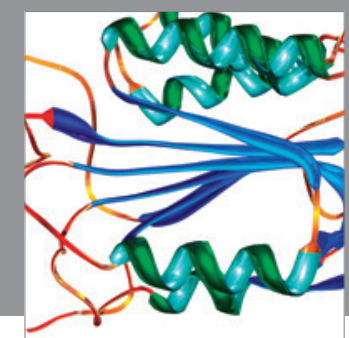

Disease Markers
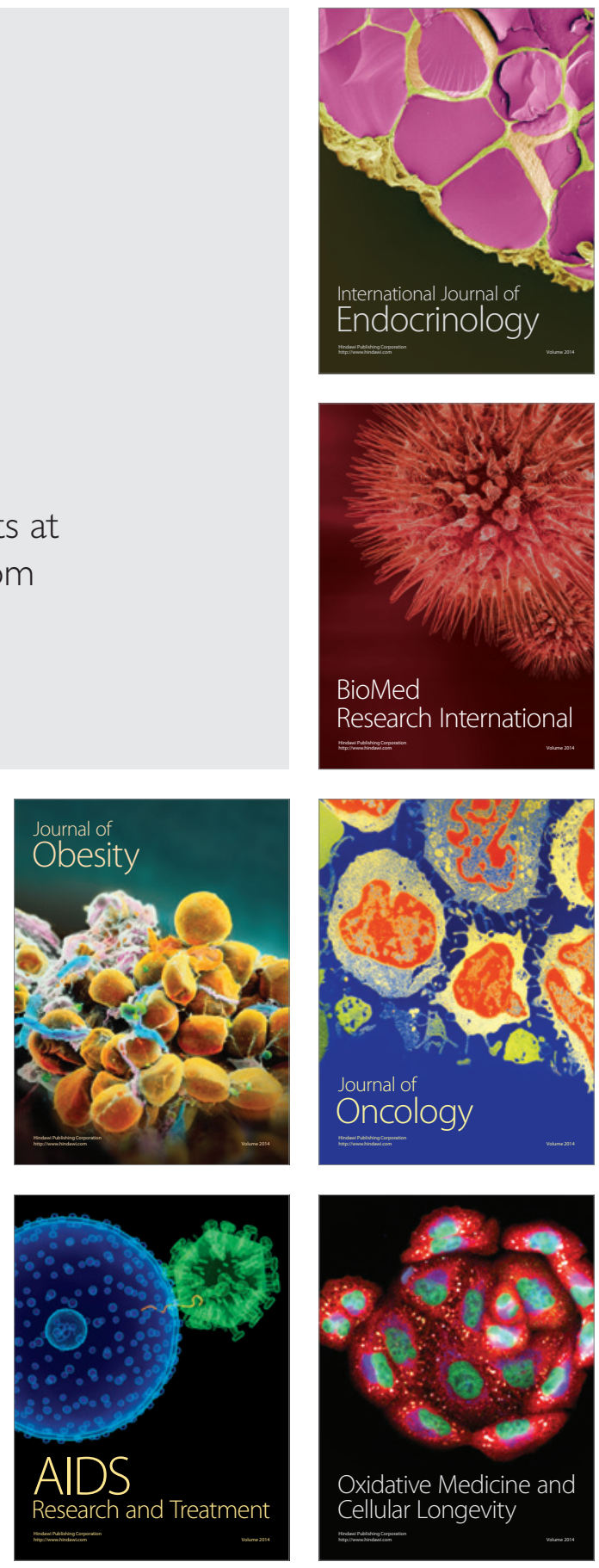